\title{
Delay Estimation for some Stationary Diffusion-type processes *
}

\author{
Uwe Küchler \\ Humboldt-Universität Berlin, Germany, \\ Yury A. Kutoyants \\ Université du Maine, Le Mans, France
}

\begin{abstract}
In this paper the asymptotic behaviour of the maximum likelihood and Bayesian estimators of a delay parameter is studied. The observed process is supposed to be the solution of a linear stochastic differential equation with one time delay term. It is shown that these estimators are consistent and their limit distributions are described. The behaviour of the estimators is similar to the behaviour of corresponding estimators in change-point problems. The question of asymptotical efficiency is also discussed.
\end{abstract}

Key words: Stochastic Differential Delay Equations, Diffusion-type process, Estimation of Delay, Asymptotic Properties for Large Sample Size, Asymptotic Efficiency

* Research for this paper was partially carried out within Sonderforschungsbereich 373. This paper was printed using funds made available by the Deutsche Forschungsgemeinschaft. 


\section{Introduction and Auxiliary Results}

In this paper we deal with the linear stochastic differential equation

$$
d X_{t}=b X_{t-\vartheta} d t+d W_{t}, \quad 0 \leq t \leq T
$$

where $b$ is a known negative parameter and the delay parameter $\vartheta$ is unknown to the observer. We have to estimate $\vartheta$ by the observation $\left\{X_{t}, 0 \leq t \leq T\right\}$. The properties of estimators will be studied in the situation of large samples: $T \rightarrow \infty$. The integral representation of the observed process

$$
X_{t}=X_{0}+\int_{0}^{t} b X_{s-\vartheta} d s+W_{t}
$$

shows that the process $X_{t}$ is as smooth with respect to time $t$ as the Wiener process $W_{t}$ therefore the trend coefficient $b X_{t-\vartheta}$ is not differentiable with respect to $\vartheta$, even the first derivative of the trend with respect to unknown parameter does not exist and the usual regularity conditions in this problem are not satisfied. The similar model of delay estimation was considered in [7] (see also [9], section 2.4, and [8]) for the observations

$$
\begin{gathered}
d X_{t}=b X_{t-\vartheta} d t+\varepsilon d W(t), \\
X(s)=x_{0}>0, s \in[-K, 0], \text { for some } K>0 \quad 0 \leq t \leq T
\end{gathered}
$$

but in asymptotics of small noise: $\varepsilon \rightarrow 0, T$ is fixed. In that problems the maximum likelihood $\hat{\vartheta}_{\varepsilon}$ and the Bayesian estimators are consistent and asymptotically normal

$$
\mathcal{L}\left\{\varepsilon^{-1}\left(\hat{\vartheta}_{\varepsilon}-\vartheta\right)\right\} \Longrightarrow \mathcal{N}(0,1)
$$

with the usual "regular" rate $\varepsilon^{-1}$ and both are asymptotically efficient. The limit variance was

$$
\sigma^{-2}=\int_{0}^{T} x_{t-2 \vartheta}^{2} d t
$$

where $x_{t}, 0 \leq t \leq T$ is the solution of the observed equation (2) with $\varepsilon=0$.

In the present work we consider the same process and the same estimators but in another asymptotics and it is in certain sense surprising that the properties of the estimators are entirely different. The maximum likelihood and Bayesian estimators have different limit distributions, the rate of convergence is $T$ (not $\sqrt{T}$ as in regular problems) and asymptotically efficient are 
the Bayesian estimators only. These results are similar to one of change-point problems see, e.g. [3], section 7.2 or [9], chapter 5. The proofs are carried out by a method of Ibragimov-Khasminski [3].

We are interested in stationary solutions of the equation (1). Their existence is given by the following

Lemma 1.1 If $b<0$ then a stationary solution of (1) exists if and only if $\vartheta \in(0,-\pi /(2 b))$.

Proof see e.g. [5], Lemma 2.9.

\section{Definitions and Results}

We observe the trajectory $X^{T}=\left\{X_{t}, 0 \leq t \leq T\right\}$ of the diffusion-type process $\left(X_{t}\right)$ given by

$$
d X_{t}=b X_{t-\vartheta} d t+d W_{t}, \quad X_{s}=X_{0}(s), \quad \frac{\pi}{2 b} \leq s \leq 0, \quad t \geq 0,
$$

where $b$ is a negative constant, $\vartheta$ is an unknown parameter from the set $\Theta=\left[0,-(e b)^{-1}\right]$ (we denote as well $\Theta_{0}=\left(0,-(e b)^{-1}\right)$ ) and $X_{0}(s)$ is a known continuous function. We are interested in asymptotic properties of the maximum likelihood and Bayes estimator of the parameter $\vartheta$ as $T \rightarrow \infty$.

Let us denote $P_{\vartheta}^{(T)}$ the measure induced in the measurable space of continuous on $[0, T]$ functions $(\mathcal{C}[0, T], \mathcal{B}[0, T])$ by the solution of the equation (3). (Here $\mathcal{B}[0, T]$ denotes the $\sigma$-algebra of Borel-subset of $\mathcal{C}[0, T]$ endowed with the supremum-norm.) The solutions of (3) corresponding to different values of $\vartheta \in \Theta$ are continuous, thus all the measures $\left\{P_{\vartheta}^{(T)}, \vartheta \in \vartheta\right\}$ are equivalent and the likelihood ratio process

$$
L\left(\vartheta, \vartheta_{1}, X^{T}\right)=\frac{d P_{\vartheta}^{(T)}}{d P_{\vartheta}^{(T)}}\left(X^{T}\right), \quad \vartheta \in \Theta
$$

is given by the formula

$$
\begin{aligned}
& L\left(\vartheta, \vartheta_{1}, X^{T}\right)= \\
& \quad=\exp \left\{b \int_{0}^{T}\left(X_{t-\vartheta}-X_{t-\vartheta_{1}}\right) d X_{t}-\frac{b^{2}}{2} \int_{0}^{T}\left(X_{t-\vartheta}^{2}-X_{t-\vartheta_{1}}^{2}\right) d t\right\},
\end{aligned}
$$


where $\vartheta_{1}$ is some fixed value (see [10]).

The maximum likelihood estimator (MLE) $\widehat{\vartheta}_{T}$ is defined as a solution of the equation

$$
L\left(\widehat{\vartheta}_{T}, \vartheta_{1}, X^{T}\right)=\sup _{\vartheta \in \Theta} L\left(\vartheta, \vartheta_{1}, X^{T}\right) .
$$

Below we suppose that the true value $\vartheta$ is an interior point of $\Theta$, i.e., $\vartheta \in \Theta_{0}$. We will see later that the function $L\left(\vartheta, \vartheta_{1}, X^{T}\right), \vartheta \in \Theta$ is continuous with probability one. Hence the MLE $\widehat{\vartheta}_{T}$ exists and belongs to $\Theta$. If the equation (4) has more than one solution than we take any one as MLE.

To introduce the Bayesian estimator we suppose that $\vartheta$ is a random variable with a prior density $\pi(y), y \in \Theta$ and the loss function is quadratic. Then the Bayes estimator (BE) $\widetilde{\vartheta}_{t}$ is a posterior mean

$$
\tilde{\vartheta}_{T}=\int_{\vartheta} y p\left(y \mid X^{T}\right) d y
$$

where the posterior density $p\left(y \mid X^{T}\right)$ is given by

$$
p\left(y \mid X^{T}\right)=\frac{\pi(y) L\left(y, \vartheta_{1}, X^{T}\right)}{\int_{\vartheta} \pi(v) L\left(v, \vartheta_{1}, X^{T}\right) d v} .
$$

The limit distributions of these estimators can be expressed through the following stochastic process

$$
Z(u)=\exp \left\{b \widetilde{W}(u)-\frac{1}{2}|u| b^{2}\right\}, \quad u \in \mathbb{R},
$$

where $\widetilde{W}(u)=W_{+}(u)$ for $u \geq 0$ and $\widetilde{W}(u)=W_{-}(-u)$ for $u<0$ and $W_{+}(u), W_{-}(u), u \geq 0$ are two independent standard Wiener processes. Let us introduce two random variables:

$$
\begin{aligned}
\xi & =\underset{u}{\arg \sup } Z(u) \\
\zeta & =\frac{\int_{-\infty}^{\infty} u Z(u) d y}{\int_{-\infty}^{\infty} Z(u) d u}
\end{aligned}
$$

The first result allows to define the asymptotically efficient estimators in this problem. 
Theorem 2.1 For any $\vartheta_{0} \in\left(0,(e b)^{-1}\right)$

$$
\lim _{\delta \rightarrow 0} \lim _{T \rightarrow \infty} \inf _{\vartheta_{T}^{*}} \sup _{\left|\vartheta-\vartheta_{0}\right|<\delta} T^{2} \mathbf{E}_{\vartheta}\left(\vartheta_{T}^{*}-\vartheta\right)^{2} \geq E \zeta^{2}
$$

Proof of this theorem follows from the theorem I.9.1 of [3] and the properties of likelihood ratio $L(\cdot)$ described below.

Therefore we can define the asymptotically efficient estimator $\vartheta_{T}^{*}$ as such an estimator, that for all $\vartheta \in \Theta_{0}$

$$
\lim _{\delta \rightarrow 0} \lim _{T \rightarrow \infty} \sup _{\left|\vartheta-\vartheta_{0}\right|<\delta} T^{2} \mathbf{E}_{\vartheta}\left(\vartheta_{T}^{*}-\vartheta\right)^{2}=E \zeta^{2}
$$

Let $\mathbf{K}$ below be an arbitrary compact set in $\Theta_{0}$. The asymptotics of MLE is given for this more narrow parameter set by the

Theorem 2.2 The MLE $\widehat{\vartheta}_{T}$ of parameter $\vartheta \in \Theta_{0}$ is uniformly in $\vartheta \in \mathbf{K}$ consistent, the normed difference $T\left(\widehat{\vartheta}_{T}-\vartheta\right)$ converges uniformly (in $\vartheta \in \mathbf{K}$ ) in distribution to the random variable $\xi$ and for any $p>0$ it holds

$$
\lim _{T \rightarrow \infty} \mathbf{E}_{\vartheta}\left|T\left(\widehat{\vartheta}_{T}-\vartheta\right)\right|^{p}=\mathrm{E}|\xi|^{p}
$$

The asymptotic behavior of the BE is better than MLE as it follows from the

Theorem 2.3 Let the prior density $\pi(y), y \in \Theta$ be a positive, continuous function, then the BE $\widetilde{\vartheta}_{T}$ is consistent in $K$ uniformly, the normed difference $T\left(\widetilde{\vartheta}_{T}-\vartheta\right)$ converges uniformly $($ in $\vartheta \in \mathbf{K}$ ) in distribution to the random variable $\zeta$ and for any $p>0$ it holds

$$
\lim _{T \rightarrow \infty} \mathrm{E}\left|T\left(\tilde{\vartheta}_{T}-\vartheta\right)\right|^{p}=\mathrm{E}|\zeta|^{p} .
$$

Moreover the BE is asymptotically efficient.

The proofs of theorems 2 and 3 is based on the general results of Ibragimov, Khasminski [3], (Theorems I.10.1, I.10.2) concerning the asymptotic behavior of MLE and BE and is given in the next section. 


\section{Proofs}

Let as introduce the normalized likelihood ratio

$$
Z_{T}(u)=\frac{d P_{\vartheta+\frac{u}{T}}^{(T)}}{d P_{\vartheta}^{(T)}}\left(X^{T}\right), \text { where } u \in U_{\vartheta, T}=\left(-\vartheta T,\left((-e b)^{-1}-\vartheta\right) T\right)
$$

and define $Z_{T}(u)$ on the intervals $[-\vartheta T-1,-\vartheta T]$ and $\left[(e b)^{-1}-\vartheta\right) T$, $\left.\left(-(e b)^{-1}-\vartheta\right) T+1\right]$ as linearly decreasing to zero. Outside the interval $\left(-\vartheta T+1,\left(-(e b)^{-1}-\vartheta\right) T+1\right)$ we put $Z_{T}(u)=0$. So the function $Z_{T}(u)$, is defined for all $u \in \mathbb{R}$ and it can be shown with the help of the lemmas 3.2 and 3.4 that it belongs to the space $\mathcal{C}_{0}$ of continuous on $\mathbb{R}$ functions decreasing to zero at infinity. Note, that

$$
T\left(\widehat{\vartheta}_{T}-\vartheta\right) \equiv \widehat{u}_{T}=\arg \max _{u} Z_{T}(u)
$$

Denote $\nu_{T}$ the measure induced in the space $\left(\mathcal{C}_{0}, \mathcal{B}_{0}\right)$ by the process $\left\{Z_{T}(u), u \in R\right\}$ and $\nu$ the measure corresponding to the process $\{Z(u), u \in$ $R\}$.

If we prove that

$$
\nu_{T} \Longrightarrow \nu
$$

then the distributions of all continuous functionals of $Z_{T}(\cdot)$ will also converge. Therefore for the functional $\Phi(z)=\sup _{u<x} z(u)-\sup _{u \geq x} z(u)$ we have

$$
\begin{aligned}
P_{\vartheta}^{(T)} & \left\{\widehat{u}_{T}<x\right\}=P_{\vartheta}^{(T)}\left\{\sup _{u<x} Z_{T}(u)>\sup _{u \geq x} Z_{T}(u)\right\}= \\
& =P_{\vartheta}^{(T)}\left\{\sup _{u<x} Z_{T}(u)-\sup _{u \geq x} Z_{T}(u)>0\right\}= \\
& =P_{\vartheta}^{(T)}\left\{\Phi\left(Z_{T}\right)>0\right\} \longrightarrow P_{\vartheta}\{\Phi(Z)>0\}= \\
& =P_{\vartheta}\left\{\sup _{u<x} Z(u)>\sup _{u \geq x} Z(u)\right\}=P_{\vartheta}\{\xi<x\} .
\end{aligned}
$$

To prove the convergence (5) we establish the convergence of finitedimentional distributions and two estimates on the increments of $Z_{T}(\cdot)$ and on the tails of $Z_{T}(\cdot)$ then according to [3], Theorems 1.10 .1 and 1.10 .2 we will have the desired properties of the MLE and BE. 
Lemma 3.1 The marginal distributions of $Z_{T}(u), u \in R$ converge to the marginal distributions of $Z(u)$ and this convergence is uniform over the compacts $\mathbf{K}$.

Proof. The likelihood ratio $Z_{T}(\cdot)$ with $P_{\vartheta}^{(T)}$ - probability one admits the representation

$$
\ln Z_{T}(u)=b \int_{0}^{T}\left(X_{t-\vartheta-\frac{u}{T}}-X_{t-\vartheta}\right) d W_{t}-\frac{b^{2}}{2} \int_{0}^{T}\left(X_{t-\vartheta-\frac{u}{T}}-X_{t-\vartheta}\right)^{2} d t .
$$

At first we show that uniformly in $\vartheta \in \mathbf{K}$

$$
\mathbf{P}_{\vartheta}-\lim _{T \rightarrow \infty} \int_{0}^{T}\left(X_{t-\vartheta-\frac{u}{T}}-X_{t-\vartheta}\right)^{2} d t=|u|,
$$

i.e., for any $\delta>0$

$$
\lim _{T \rightarrow \infty} \sup _{\vartheta \in \mathbf{K}} \mathbf{P}_{\vartheta}^{(T)}\left\{\left|\int_{0}^{T}\left(X_{t-\vartheta-\frac{u}{T}}-X_{t-\vartheta}\right)^{2} d t-\right| u||>\delta\right\}=0 .
$$

Let $u>0$. The difference $Y_{t}(u)=X_{t-\vartheta-\frac{u}{T}}-X_{t-\vartheta}$ can be written as

$$
\begin{array}{r}
Y_{t}(u)=b \int_{t-\vartheta}^{t-\vartheta-\frac{u}{T}} X_{s-\vartheta} d s+W_{t-\vartheta-\frac{u}{T}}-W_{t-\vartheta}= \\
=\frac{b u}{T} X_{t-2 \vartheta}(1+o(1))+\frac{1}{\sqrt{T}} \Delta_{t}(u) .
\end{array}
$$

Here

$$
\Delta_{t}(u)=T^{1 / 2}\left(W_{t-\vartheta-\frac{u}{T}}-W_{t-\vartheta}\right)
$$

is a Wiener process with respect to $u \geq 0$ :

$$
\mathbf{E} \Delta_{t}(u)=0, \quad \mathbf{E} \Delta_{t}(u)^{2}=u, \quad \mathbf{E} \Delta_{t}(u) \Delta_{t}(v)=\min (u, v) .
$$

Therefore

$$
Y_{t}(u)=\frac{1}{\sqrt{T}} \Delta_{t}(u)(1+o(1))
$$


because the process $X_{t}$ is Gaussian ergodic and $T^{-1 / 2} X_{t} \rightarrow 0$ as well as $T^{-1 / 2} \mathbf{E}_{\vartheta}\left|X_{t}\right|^{p} \rightarrow 0, p>0$ as $T \rightarrow \infty$. We can write

$$
\begin{aligned}
\sup _{\vartheta \in \mathbf{K}} & \mathbf{E}_{\vartheta}\left(\int_{0}^{T} Y_{t}(u)^{2} d t-|u|\right)^{2}= \\
& =\sup _{\vartheta \in \mathbf{K}} \mathbf{E}_{\vartheta}\left(\frac{1}{T} \int_{0}^{T}\left[\Delta_{t}(u)^{2}-|u|\right] d t\right)^{2}(1+o(1)) \leq \\
& \leq \frac{1}{T^{2}} \int_{0}^{T} \int_{0}^{T} \sup _{\vartheta \in \mathbf{K}} \mathbf{E}_{\vartheta}\left(\Delta_{t}(u)^{2} \Delta_{s}(u)^{2}-u^{2}\right)(1+o(1)) d s d t .
\end{aligned}
$$

For the values $s, t$ such that $|t-s|>|u| / T$ these increments of the Wiener process are independent and we have

$$
\mathbf{E}_{\vartheta} \Delta_{t}(u)^{2} \Delta_{s}(u)^{2}=u^{2} .
$$

In the case $|t-s| \leq|u| / T$, say, $t<s$ we have $s-u / T<t$ and using independence of the increments $W_{s}-W_{t}, W_{t-u / T}-W_{t}, W_{t-u / T}-W_{s-u / T}$ we can write

$$
\mathbf{E}_{\vartheta} \Delta_{t}(u)^{2} \Delta_{s}(u)^{2}-u^{2} \leq 6 u^{2}
$$

Therefore

$$
\sup _{\vartheta \in \Theta} \int_{0}^{T} \int_{0}^{T} \mathbf{E}_{\vartheta}\left(\left[Y_{t}(u)^{2}-|u| / T\right]\left[Y_{s}(u)^{2}-|u| / T\right]\right) d t d s \leq C \frac{u^{2}}{T}
$$

The similar arguments allow to prove the uniform convergence

$$
\mathbf{P}_{\vartheta}-\lim _{T \rightarrow \infty} \int_{0}^{T} Y_{t}(u) Y_{t}(v) d t=|u| \wedge|v|
$$

The central limit theorem for stochastic integrals ([6], Theorem 3.3.7) and convergence (6) provide the uniform asymptotic normality

$$
\mathcal{L}\left\{\int_{0}^{T} Y_{t}(u) d W_{t}\right\} \Longrightarrow \mathcal{N}(0,|u|) .
$$

The same theorem and condition (7) provide the convergence of the finitedimensional distributions of the process

$$
z_{T}(u)=\int_{0}^{T} Y_{t}(u) d W_{t}
$$

to the finite dimensional distributions of the process $\widetilde{W}(u), u \in \mathbb{R}$. 
Lemma 3.2 There exists a constant $\mathbf{C}_{0}$ such that for any $R>0$

$$
\sup _{\vartheta \in \mathbf{K}} \sup _{|v|<R,|u|<R}|u-v|^{-2} \mathbf{E}_{\vartheta}\left|Z_{T}^{\frac{1}{4}}(u)-Z_{T}^{\frac{1}{4}}(v)\right|^{4} \leq \mathbf{C}_{0}\left(1+R^{6}\right)
$$

Proof is similar to the proof of the Lemma 2.6 in [9]. Let us denote

$$
V(T)=\left(\frac{d \mathbf{P}_{\vartheta+u / T}^{(T)}}{d \mathbf{P}_{\vartheta+v / T}^{(T)}}\left(X_{T}\right)\right)^{\frac{1}{4}}
$$

Then by Itô's formula with $\mathbf{P}_{\vartheta+u_{1 / T}}^{(T)}$-probability one

$$
\begin{aligned}
V(T)=1 & -\frac{3}{32} b^{2} \int_{0}^{T} V(t)\left[X_{t-\vartheta-\frac{u}{T}}-X_{t-\vartheta-\frac{v}{T}}\right]^{2} d t+ \\
& +\frac{b}{4} \int_{0}^{T} V(t)\left[X_{t-\vartheta-\frac{u}{T}}-X_{t-\vartheta-\frac{v}{T}}\right] d W_{t}
\end{aligned}
$$

Therefore denoting $Y_{t}(u, v)=X_{t-\vartheta-\frac{u}{T}}-X_{t-\vartheta-\frac{v}{T}}$ we can write

$$
\begin{aligned}
& \mathbf{E}_{\vartheta}\left|Z_{T}^{\frac{1}{4}}(u)-Z_{T}^{\frac{1}{4}}(v)\right|^{4}=\mathbf{E}_{\vartheta} Z_{T}(v)|V(T)-1|^{4}= \\
& =\mathbf{E}_{\vartheta+v / T}\left|-\frac{3 b^{2}}{32} \int_{0}^{T} V(t) Y_{t}(u, v)^{2} d t+\frac{b}{4} \int_{0}^{T} V(t) Y_{t}(u, v) d W_{t}\right|^{4} \leq \\
& \leq C_{1} b^{8} T^{3} \int_{0}^{T} \mathbf{E}_{\vartheta+v / T} V(t)^{4} Y_{t}(u, v)^{8} d t+ \\
& \quad+C_{2} b^{4} T \int_{0}^{T} \mathbf{E}_{\vartheta+v / T} V(t)^{4} Y_{t}(u, v)^{4} d t= \\
& =C_{1} b^{8} T^{3} \int_{0}^{T} \mathbf{E}_{\vartheta+u / T} Y_{t}(u, v)^{8} d t+C_{2} b^{4} T \int_{0}^{T} \mathbf{E}_{\vartheta+u / T} Y_{t}(u, v)^{4} d t .
\end{aligned}
$$

The moments of the process $Y_{t}(u, v)$ can be estimated as follows

$$
\begin{aligned}
& \mathbf{E}_{\vartheta+u / T} Y_{t}(u, v)^{8}= \\
& =\mathbf{E}_{\vartheta+u / T}\left(b \int_{t-\vartheta-v / T}^{t-\vartheta-u / T} X_{s-\vartheta-u / T} d s+W_{t-\vartheta-v / T}-W_{t-\vartheta-u / T}\right)^{8} \leq \\
& \leq C_{3} b^{8}\left|\frac{u-v}{T}\right|^{7} \int_{t-\vartheta-v / T}^{t-\vartheta-u / T} \mathbf{E}_{\vartheta+u / T} X_{s-\vartheta-u / T}^{8} d s+C_{4}\left|\frac{u-v}{T}\right|^{4} \leq \\
& \leq C_{5}\left|\frac{u-v}{T}\right|^{8}+C_{6}\left|\frac{u-v}{T}\right|^{4}
\end{aligned}
$$


For the second integral similar calculations provide

$$
\mathbf{E}_{\vartheta+u / T} Y_{t}(u, v)^{4} \leq C_{7}\left|\frac{u-v}{T}\right|^{4}+C_{8}\left|\frac{u-v}{T}\right|^{2}
$$

Therefore we have

$$
\begin{aligned}
& \mathbf{E}_{\vartheta}\left|Z_{T}^{\frac{1}{4}}(u)-Z_{T}^{\frac{1}{4}}(v)\right|^{4} \leq C_{9}|u-v|^{8}+C_{10}|u-v|^{4}+C_{11}|u-v|^{2} \leq \\
& \quad \leq C_{0}\left(1+R^{6}\right)|u-v|^{2}
\end{aligned}
$$

where the constant $C_{0}>0$ does not depend on $R$ and $\vartheta$.

We shall need the following estimate.

Lemma 3.3 Let $Y_{t}, 0 \leq t \leq T$ be a mean square integrable zero mean Gaussian process with covariance $R(t, s)$ then

$$
\mathbf{E} \exp \left\{-\int_{0}^{T} Y_{t}^{2} d t\right\} \leq \exp \left\{-\frac{\operatorname{tr}(R)}{1+2\|R\|}\right\}
$$

where

$$
\operatorname{tr}(R)=\int_{0}^{T} R(t, t) d t, \quad\|R\|^{2}=\int_{0}^{T} \int_{0}^{T} R(t, s)^{2} d t d s .
$$

Proof see Kallianpur and Selukar [4], Lemma 3.1.

Lemma 3.4 There exist constants $\kappa=\kappa(\mathbf{K})>0$ and $\mathbf{C}_{1}$ such that

$$
\sup _{\vartheta \in \mathbf{K}} \mathbf{E}_{\vartheta} Z_{T}^{\frac{1}{2}}(u) \leq \mathbf{C}_{1} \mathrm{e}^{-\kappa\left(|u| \wedge|2 b u|^{1 / 2}\right)}
$$

Proof. First we obtain the estimate

$$
\mathbf{E}_{\vartheta} Z_{T}^{1 / 2}(u) \leq\left\{\mathbf{E}_{\vartheta} \exp \left(-\frac{1}{8} \int_{0}^{T} Y_{t}(u)^{2} d t\right)\right\}^{1 / 2}
$$

(see [6], Lemma 3.4.3, or [4], Lemma 3.4) and then using (8) write the inequality

$$
\mathbf{E}_{\vartheta} Z_{T}^{1 / 2}(u) \leq \exp \left\{-\frac{1}{16} \frac{\operatorname{tr}(R)}{1+2\|R\|}\right\}
$$


where $Y_{t}(u)=X_{t-\vartheta-u / T}-X_{t-\vartheta}$. The process $X_{t}, t \geq 0$ can be written in the following form

$$
X_{t}=x_{0}(t) X_{0}(0)+b \int_{-r}^{t} x_{0}(t-s-r) X_{0}(s) \mathrm{d} s+\int_{0}^{t} x_{0}(t-s) \mathrm{d} W_{s}
$$

where $x_{0}($.$) the fundamental solution of the corresponding to (1) determin-$ istic equation

$$
\frac{x_{0}(t)}{d t}=b x_{0}(t-\vartheta), \quad x_{0}(s)=\mathbf{I}_{\{0\}}(s), s \leq 0
$$

(see Küchler, Mensch [5], Proposition 2.2). Moreover for some $\gamma>0$ the estimate

$$
\left|x_{0}(t)\right| \leq K_{0} e^{-\gamma t}, \quad t \geq 0
$$

is valid. The stochastic process $X_{t}, t \geq 0$ admits as well the representation $X_{t}=U_{t}+R_{t}, t \geq 0$ where $U_{t}, t \geq 0$ is the stationary part, i.e., zero mean stationary Gaussian process with the covariance

$$
K(t-s)=\int_{0}^{\infty} x_{0}(|t-s|+v) x_{0}(v) \mathrm{d} v
$$

and $\mathbf{E} R_{t}^{2} \leq K_{1} e^{-\gamma t}$ (see [5], (2.19)). Therefore in the estimates below it is sufficient to consider the stationary part only. Indeed we have for the function $R_{u}(t, s)=\mathbf{E}_{\vartheta} Y_{t}(u) Y_{s}(u)$ the estimate

$$
\left|R_{u}(t, s)-2 K(t-s)+K(t-s+u / T)+K(t-s-u / T)\right| \leq K e^{-\gamma(t+s)} .
$$

Note that for $b<0$ and $\vartheta \leq-(e b)^{-1}$ the solution of the equation (10) is positive for all $t \geq 0$ see, e.g., [2] or [5], Lemma 3.3. Therefore

$$
\operatorname{tr}\left(R_{u}\right)=2 T\left[K(0)-K\left(\frac{u}{T}\right)\right] .
$$

Introduce the function

$$
G(\alpha)=\int_{0}^{\infty}\left[x_{0}(s)^{2}-x_{0}(s) x_{0}(s+\alpha)\right] d s
$$

The derivative of this function is

$$
\dot{G}(\alpha)=-\int_{0}^{\infty} x_{0}(s) \dot{x}_{0}(s+\alpha) d s=-b \int_{0}^{\infty} x_{0}(s) x_{0}(s+\alpha-\vartheta) d s>0
$$


because the function $x_{0}(s)$ is positive for all $s \geq 0$ and the set $\Theta$ is compact. Therefore there exist constants $\kappa_{1}>0, a>0$ such that

$$
\operatorname{tr}\left(R_{u}\right) \geq \kappa_{1}|u|-a .
$$

Further, the function $K(t)$ satisfies the same equation as $x_{0}(s)$ (see [5], (2.23)) therefore for this function by Lemma 2.5 of [5] we have the estimates

$$
|K(t)| \leq K_{0} e^{-\gamma|t|}, \quad|\dot{K}(t)| \leq K_{1} e^{-\gamma|t|}
$$

where $K_{0}, K_{1}$ and $\gamma=\gamma(\mathbf{K})$ some positive constants which can be chosen not depending on delay $\vartheta \in \mathbf{K}$.

We have for $|u| \geq 1$ and for some $c>0$

$$
\begin{aligned}
\int_{0}^{T} & \int_{0}^{T} R_{u}(t, s)^{2} d t d s \leq \\
& \leq \int_{0}^{T} \int_{0}^{T}\left[2 K(t-s)-K\left(t-s-\frac{u}{T}\right)-K\left(t-s+\frac{u}{T}\right)\right]^{2} d t d s+c= \\
& =2 T \int_{0}^{T}\left[2 K(t)-K\left(t-\frac{u}{T}\right)-K\left(t+\frac{u}{T}\right)\right]^{2} d t+c \leq \\
& \leq \frac{8 u^{2}}{T} \int_{0}^{T} \dot{K}\left(t-\frac{\tilde{u}}{T}\right)^{2} d t+c \leq C^{2} \frac{u^{2}}{4 T}
\end{aligned}
$$

Therefore

and we obtain the estimate

$$
\left\|R_{u}\right\| \leq \frac{C|u|}{2 \sqrt{T}}
$$

$$
\frac{\operatorname{tr}\left(R_{u}\right)}{1+2\left\|R_{u}\right\|} \geq \frac{\kappa_{1}|u|}{1+C T^{-1 / 2}|u|} .
$$

If $|u|<\sqrt{T}$ then we have

$$
\frac{\kappa_{1}|u|}{1+C T^{-1 / 2}|u|} \geq \frac{\kappa_{1}}{1+C}|u| .
$$

If $|u| \geq \sqrt{T}$ then we can write

$$
\begin{aligned}
\frac{\kappa_{1}|u|}{1+C T^{-1 / 2}|u|} & =\frac{\kappa_{1}}{C} T^{1 / 2}-\frac{\kappa_{1} T^{1 / 2}}{C\left(1+C T^{-1 / 2}|u|\right)} \geq \\
& \geq \frac{\kappa_{1}}{C} T^{1 / 2}-\frac{\kappa_{1} T^{1 / 2}}{C(1+C)}= \\
& =\frac{\kappa_{1}}{(1+C)} T^{1 / 2} \geq \frac{\kappa_{1}}{(1+C)}(-b e)^{1 / 2}|u|^{1 / 2}
\end{aligned}
$$


because for all $u$ we have the obvious estimate $|u|<T\left(-(e b)^{-1}\right)$.

Hence we obtained (9) with corresponding $\kappa>0$ and $\mathbf{C}_{1}$.

The properties of the likelihood ratio established above allow to use the Theorems 1.10 .1 and 1.10.2 of [3] to finish the proofs of the Theorems 2.2 and 2.3 above.

The proof of the Theorem 2.1 follows from the properties of the Bayes estimators established above and the Theorem 1.9.1 in [3].

\section{References}

[1] Gushchin, A. \& Küchler, U. (1997). Asymptotic Inference for a Linear Stochastic Differential Equation with Time Delay, to appear in Bernoulli, Discussion Paper 43(1997) of Sonderforschungsbereich 373, Humboldt University Berlin.

[2] Hale, J.K. \& Verduyn Lunel, S.M. (1993). Introduction to FunctionalDifferential Equations. Springer, New York.

[3] Ibragimov, I.A. \& Khasminskii, R.Z. (1981). Statistical Estimation. Springer, Berlin.

[4] Kallianpur, G. \& Selukar, R.S. (1991). Parameter estimation in linear filtering. J. Multivar. Analysis, 39, 284-304.

[5] Küchler, U. \& Mensch, B. (1991). Langevins stochastic differential equation extended by a time-delayed term. Stochastics and Stochastic Reports, 40, 23-42.

[6] Kutoyants, Yu.A. (1984). Parameter Estimation for Stochastic Processes, Heldermann, Berlin.

[7] Kutoyants, Yu.A. (1988). An example of estimating a parameter of a nondifferentiable drift, Theory Probab. Appl., 33, 1, 175-179.

[8] Kutoyants, Yu.A., Mourid, T. \& Bosq, D. (1992). Estimation paramétrique d'un processus de diffusion avec retard, Ann. Inst. Henry Poincaré, 28, 1, 95-106. 
[9] Kutoyants, Yu.A. (1994). Identification of Dynamical systems with Small Noise, Kluwer, Dordrecht.

[10] Liptser, R.S. \& Shiryayev, A.N. (1977). Statistics of Random Processes, Springer, Berlin.

[11] Mohammed, S.-E.A. and Scheutzow, M.K.R. (1990). Lyapunov exponents and stationary solutions for affine stochastic delay equations. Stochastics and Stochastic Reports, 29, 259-283. 